\title{
Ultrasonic Scattered Field Distribution of One and Two Cylindrical Solids with Phased Array Technique
}

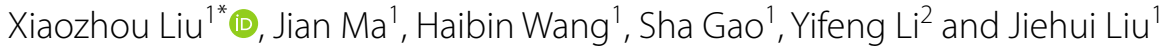

\begin{abstract}
The scattered fields of plane waves in a solid from a cylinder or sphere are critical in determining its acoustic characteristics as well as in engineering applications. This paper investigates the scattered field distributions of different incident waves created by elastic cylinders embedded in an elastic isotropic medium. Scattered waves, including longitudinal and transverse waves both inside and outside the cylinder, are described with specific modalities under an incident plane wave. A model with a scatterer embedded in a structural steel matrix and filled with aluminum is developed for comparison with the theoretical solution. The frequency of the plane wave ranged from $235 \mathrm{kHz}$ to $2348 \mathrm{kHz}$, which corresponds to scaling factors from 0.5 to 5 . Scattered field distributions in matrix materials blocked by an elastic cylindrical solid have been obtained by simulation or calculated using existing parameters. The simulation results are in good agreement with the theoretical solution, which supports the correctness of the simulation analysis. Furthermore, ultrasonic phased arrays are used to study scattered fields by changing the characteristics of the incident wave. On this foundation, a partial preliminary study of the scattered field distribution of double cylinders in a solid has been carried out, and the scattered field distribution at a given distance has been found to exhibit particular behaviors at different moments. Further studies on directivities and scattered fields are expected to improve the quantification of scattered images in isotropic solid materials by the phased array technique.
\end{abstract}

Keywords: Ultrasonic phased array, Scattered field distribution, Directivity, Quantification of scattered image

\section{Introduction}

The theoretical problem of ultrasonic scattering from a cylindrical or spherical obstacle has been studied by many researchers in the past several decades. In the early 1950s, an eigenvalue mode expansion method was proposed, and the scattered field of an elastic cylinder immersed in a fluid was first analyzed theoretically and experimentally [1]. The characteristics of the peripheral waves from plane wave scattering by a circular cylindrical shell were analyzed and compared with calculated results [2]. The scattering problems of a plane wave were also discussed under various conditions [3-6]. Theoretical predictions have also been widely verified by laboratory

\footnotetext{
*Correspondence: xzliu@nju.edu.cn

${ }^{1}$ Key Laboratory of Modern Acoustics, Institute of Acoustics and School of Physics, Nanjing University, Nanjing 210093, China

Full list of author information is available at the end of the article
}

measurements and studies [7, 8]. A system with a frequency $(f)$ range of $0<f<100 \mathrm{MHz}$ (equivalent to $0<k a<7.2$ ) reached good agreement for $2.5<k a<6.5$ on some parts of a fiber [9].

The corresponding problem for a solid elastic scatterer embedded in an elastic matrix has also attracted research attention. A general physical model was proposed for acoustic scattering from heterogeneous cylinders in solids, which described the scattering characteristics of both plane compressive and shear waves incident upon a solid cylindrical discontinuity in an isotropic solid [10]. This work used three displacement potential functions to describe the scattered wave equations for an elastic solid cylinder and derived the scattering equations for a fluidfilled cylindrical bore, which are similar to the displacement potential functions of a bulk wave in classical Biot theory [11-14]. The stress distribution at the interface of the elastic scattering cylinder was calculated in detail 
based on the theory of solid mechanics [15]. Assuming mode conversion in a solid, both scattered longitudinal waves and scattered shear waves were generated simultaneously [16]. Acoustic wave attenuation in the medium was taken into consideration and applied to the scattered field for a cylindrical cavity in an elastic medium $[17,18]$. Attenuation was found to be universal, depending on the components and interface of the medium [19, 20], and the scattering displacement field was recalculated based on the time-domain impulse responses under this condition.

The methods for dealing with scattered fields for spheres and cylinders are interlinked in some respects. The problem of scattering from solid spheres in an elastic isotropic solid was first examined theoretically in 1956 [21]. A resonant scattering theory analysis was later presented for a Lucite sphere with an iron spherical inclusion [22]. The resonances of an elastic sphere embedded in an elastic isotropic medium and the dispersion curves for the phase velocities of circumferential waves were also obtained [23]. In fact, each scattering problem involving spherical solid inclusions in solid matrices appeared to be derived from almost the same theoretical foundation [24-28], and today's more extensive scattering fields have made some progress, such as large deformation shape [29], corners and circular cones [30], time-harmonic field $[31,32]$, and poroelastic extension and single negative metamaterials [33, 34].

Ultrasonic phased array inspection technology can detect complex geometric components using ultrasonic beams. The deflection beam and focus beam of a phased array are normally generated with a time delay, which ensures a larger detection region for the sample [35]. The phased array technique can measure the scattering matrix of the defects and extract their signal characteristics, but the specific inconsistency of each array element has been found to have a great influence on defect detection and imaging [36, 37]. The angular distribution of a scattered field in a solid or saturated fluid has been discussed by many researchers [10, 38]. However, the main challenge is to improve the quantification of the scattered image obtained experimentally by the phased array technique, which is already used in industry. Ultrasonic phased array systems have always been used to measure and image a sample rather than obtain quantitative scattered pressure or directivity distributions. The focus of this paper is the scattered fields of one and two cylindrical inclusions obtained using the phased array technique, which will help provide some theoretical support for improving phased arrays for quantitative nondestructive testing.

The remainder of this paper is organized as follows. Section 2 presents the scattering theory of plane wave incidence in an elastic and isotropic medium. The displacement potentials in the matrix and the scatterer are respectively established, after which the scattering coefficients are obtained from equations according to boundary conditions. A theoretical and numerical simulation with different frequencies are then obtained in Section 3. Then, application of the phased array technique to the scattered pressure distribution, as well as to the directivity of single and double cylinders, is described. The paper ends with conclusions and a description of future work in Section 4.

\section{Theory and Model}

\subsection{Scattering Theory of Plane Wave Incidence}

The theory of acoustic fields and wave propagation in elastic solids is relatively mature [15]. Many plane longitudinal waves propagating in an elastic medium with density $\rho_{1}$ and velocity $c_{1 L}$ are vertically incident on an infinitely long elastic cylinder with density $\rho_{2}$ and velocity $c_{2 L}$, as illustrated in Figure 1. Note that the solid elastic matrix is labeled as material 1 and the solid cylinder as material 2. Due to mode conversion at the interface between the two solids, scattered compressive and shear waves are produced in material 1. $r$ is the radial distance from the center of the inclusion, and $\theta$ is the angle measured counterclockwise from the positive $x$-axis, as shown in Figure 1. A plane wave in the incident field can be written in the form of a displacement potential:

$$
\Phi_{i}=\Phi_{0} e^{i(k r \cos \theta-\omega t)}
$$

where $\Phi_{0}$ is the incident amplitude, $k=\omega / c$ is the wavenumber, $\omega$ is the angular frequency, $c$ is the velocity in the material, and $t$ is the time of wave propagation. For convenience in calculation, the incident amplitude $\Phi_{0}$ is set to one, and the time factor is omitted.

It is very difficult to solve Eq. (1) directly. Inspired by the boundary conditions at the cylinder surface, the plane wave, which is decomposed into an infinite sum

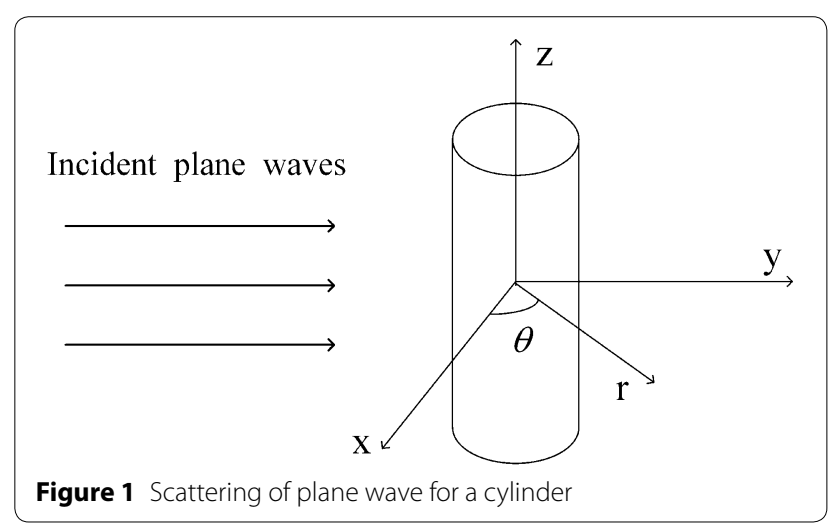


of cylindrical Bessel functions, provides a solution. The incident potential can be written as

$$
\Phi_{i}=\sum_{n=0}^{\infty} i^{n} \varepsilon_{n} J_{n}\left(k_{1 L} r\right) \cos (n \varphi),
$$

where $\Phi_{\iota}$ has been normalized to unity, $k_{1 L}$ is the wavenumber, which is equal to $\omega / c_{1 L}$ in material $1, J_{n}$ is the cylindrical Bessel function of the first kind of order $n$, and $\varepsilon_{n}$ is the Neumann coefficient.

In addition to the mode conversion occurring in material 1, longitudinal and transverse waves are also excited in material 2 . Similarly, they can be represented by displacement potentials [18]:

$$
\begin{aligned}
& \Phi_{1 L}=\sum_{n=0}^{\infty} i^{n} \varepsilon_{n} A_{n} H_{n}^{(1)}\left(k_{1 L} r\right) \cos (n \varphi), \\
& \Phi_{1 S}=\sum_{n=0}^{\infty} i^{n} \varepsilon_{n} B_{n} H_{n}^{(1)}\left(k_{1 S} r\right) \sin (n \varphi), \\
& \Phi_{2 L}=\sum_{n=0}^{\infty} i^{n} \varepsilon_{n} C_{n} J_{n}\left(k_{2 L} r\right) \cos (n \varphi), \\
& \Phi_{2 S}=\sum_{n=0}^{\infty} i^{n} \varepsilon_{n} D_{n} J_{n}\left(k_{2 S} r\right) \sin (n \varphi),
\end{aligned}
$$

where $\Phi_{1 L}$ represents the compressive wave potential, $\Phi_{1 S}$ the shear wave potential in material 1 , and definitions are analogous for material 2. $H_{n}^{(1)}$ is a cylindrical Hankel function of the first kind, $A_{n}$ and $B_{n}$ are undetermined coefficients in material 1 , and $C_{n}$ and $D_{n}$ are undetermined coefficients in material 2. $k_{1 L}$ and $k_{1 S}$ are compressive and shear wavenumbers in material 1 , respectively, and $k_{2 L}$ and $k_{2 S}$ are the same in material 2.

At the surface separating two elastic media, the conditions are easily derivable. At such a surface, it is necessary to require that the two media remain in perfect contact, which leads to the conditions that the displacements must be continuous across the boundary and that an arbitrary volume enclosing portions of both media be at equilibrium, which leads to continuity of normal traction across the cylindrical surface. The boundary conditions for stress and displacement have specific expression forms.

Note that the field outside the cylinder is the sum of the incident and scattered fields, whereas the field inside the cylinder is only the transmitted field. Combined with the theory of elastic media, a matrix equation involving the coefficients $A_{n}, B_{n}, C_{n}, D_{n}$ can be obtained [16]:

$$
\left|a_{i, j}^{(n)}\right|\left|A_{n}, B_{n}, C_{n}, D_{n}\right|^{\mathrm{T}}=\left|F_{i n}\right|,
$$

where $i, j=1,2,3$ and 4 . The expressions for each element $a_{i, j}^{(n)}$ and $F_{i n}$ can be calculated using the material parameters described above. Hence, the undetermined coefficients for waves inside and outside the cylinder can be calculated by the matrix equation, and then the scattered wave potentials can be obtained.

\subsection{Simulation Model}

The goal of the simulation model is to verify the results of theoretical calculations using ultrasonic scattered field data. The simulation geometry, a two-dimensional model, consists of a rectangular matrix and a cylindrical scatterer filled with different materials. The medium is $140 \mathrm{~mm}$ wide and $50 \mathrm{~mm}$ deep. The coordinate origin is located in the center of the rectangle on the edge. The center of the scatterer lies at 0 to $25 \mathrm{~mm}$, which ensures that the scatterer generates a symmetric spatial distribution. The length of the cylinder is infinite, and the bottom radius of the cylinder is $2 \mathrm{~mm}$.

To perform the calculations, a simulation model has been developed by the authors using the finite element method $[39,40]$. The program uses the theory of elastic wave propagation in a solid. The input signal is located at the top of the rectangular matrix, just above the cylindrical scatterer. A plane wave and Gaussian phased array signal are used as input signals to excite the solid to generate various constituent waves.

\section{Numerical Simulations \\ 3.1 Theoretical Verification of Scattered Longitudinal Waves}

In this study, which involves modeling the propagation of a longitudinal wave in an isotropic medium, the focus is on observation of scattered longitudinal and transverse waves and the angular distribution of scattered waves in relation to the frequency of incident waves in the medium. This characteristic in relation to the $k a$ product makes it possible to observe frequency behaviors according to the scatterer in the medium. The tentative matrix is assumed to be non-dispersive and non-attenuating, a behavior that is linked to the appearance of scattering.

To verify this theory, structural steel and aluminum are used as the matrix and scatterer materials, respectively. Table 1 lists their parameters in detail.

Figure 2 shows a set of numerical simulations describing the angular scattered pressure distribution of an incident plane wave. The rectangular matrix medium is excited on the top using a plane wave as the vertical velocity input. Acoustic pressure data are collected by probes on the cylindrical surface. The results give the 
Table 1 Material properties of the simulation model

\begin{tabular}{llllll}
\hline Material & $\begin{array}{l}\text { Longitudinal } \\
\text { wave } \\
\text { velocity } \\
\boldsymbol{v}_{L}(\mathbf{m} / \mathbf{s})\end{array}$ & $\begin{array}{l}\text { Transverse } \\
\text { wave } \\
\text { velocity } \\
\boldsymbol{v}_{S}(\mathbf{m} / \mathbf{s})\end{array}$ & $\begin{array}{l}\text { Density } \\
\left(\mathbf{k g} / \mathbf{m}^{3}\right)\end{array}$ & $\begin{array}{l}\text { Lamé } \\
\boldsymbol{\lambda}\end{array}$ & $\begin{array}{l}\text { Lamé } \\
\boldsymbol{\mu}\end{array}$ \\
\hline $\begin{array}{c}\text { Alu- } \\
\text { minum } \\
\text { Steel }\end{array}$ & 6568 & 3149 & 2700 & $5.1 \times 10^{10}$ & $2.6 \times 10^{10}$ \\
\hline
\end{tabular}

angular scattered pressure distribution as a function of $k a$ at four frequency levels: 225, 850, 1409, and $2248 \mathrm{kHz}$, corresponding to the four cases shown in Figure 2. The angular distribution of the scattered field in Figure 2a is almost invariant, which indicates that when the frequency is relatively low, the presence of the scatterer has little influence on the scattered pressure amplitude. As the frequency increases, the scatterer presents an obvious obstruction to the incident wave, and backscattering is fairly intense, as shown in Figure 3b. When the frequency is high enough, the scattered waves will gradually disperse, and a series of sidelobes will appear, as shown in Figure $2 \mathrm{c}$ and $\mathrm{d}$. The higher the frequency of the incident plane wave, the larger the scattered pressure amplitude will be, and the greater the number of sidelobes generated will be. Graphically, although the simulation results are always less than the theoretical solutions, they are

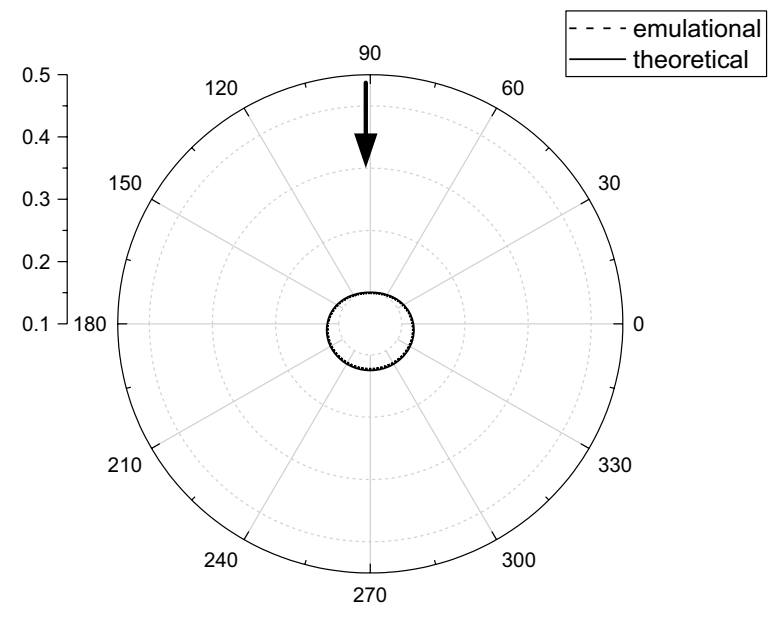

$\mathbf{a}$

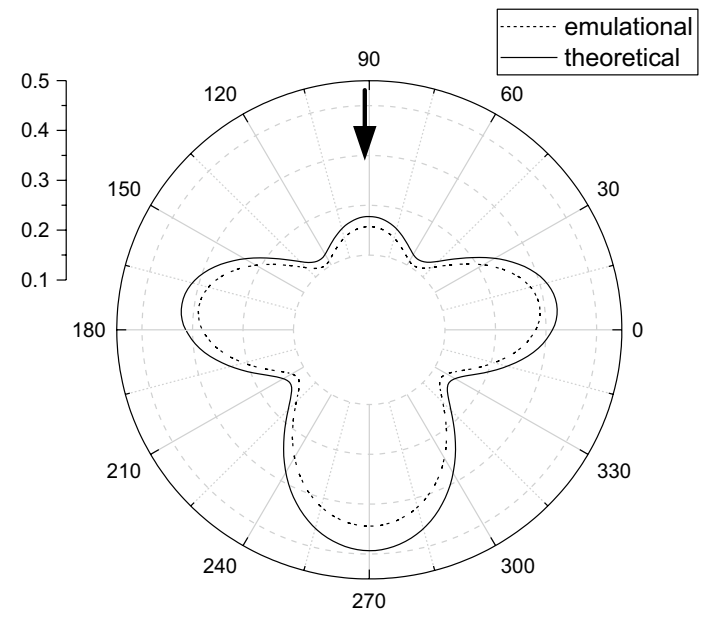

c

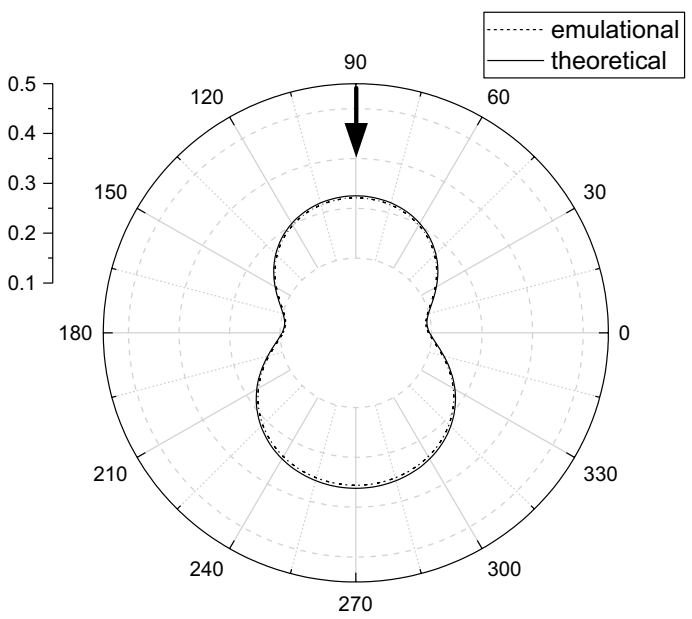

b

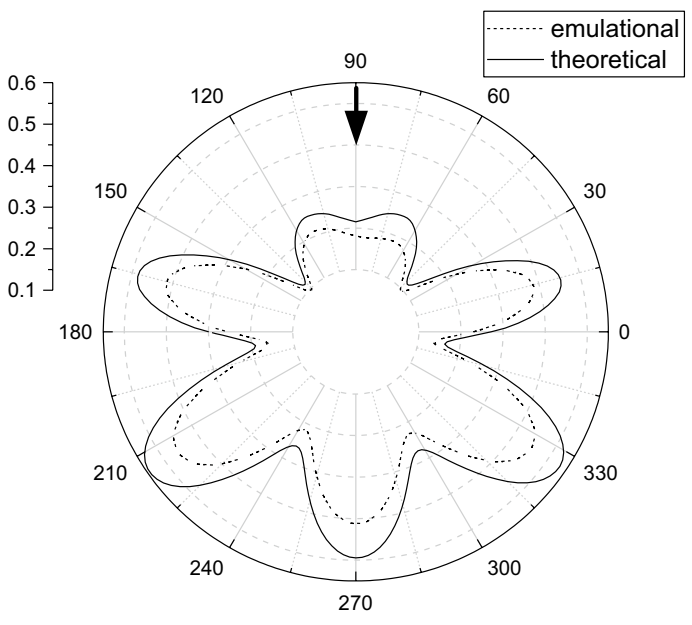

d

Figure 2 Angular distribution of scattered pressure of plane wave from simulation results and theoretical solutions versus $k a(f=235 \mathrm{kHz}, 850 \mathrm{kHz}$ $1109 \mathrm{kHz}$, and $2348 \mathrm{kHz}$ ) incident pressure amplitude is one, plane wave width is $2.8 \mathrm{~mm}$, and cylinder radius is $2 \mathrm{~mm}$ ): $\mathbf{a} \mathrm{ka}=0.5, \mathbf{b} \mathrm{ka}=1.8, \mathbf{c}$ $k a=3.0$, and $\mathbf{d} k a=5.0$. The arrows represent the plane wave direction 


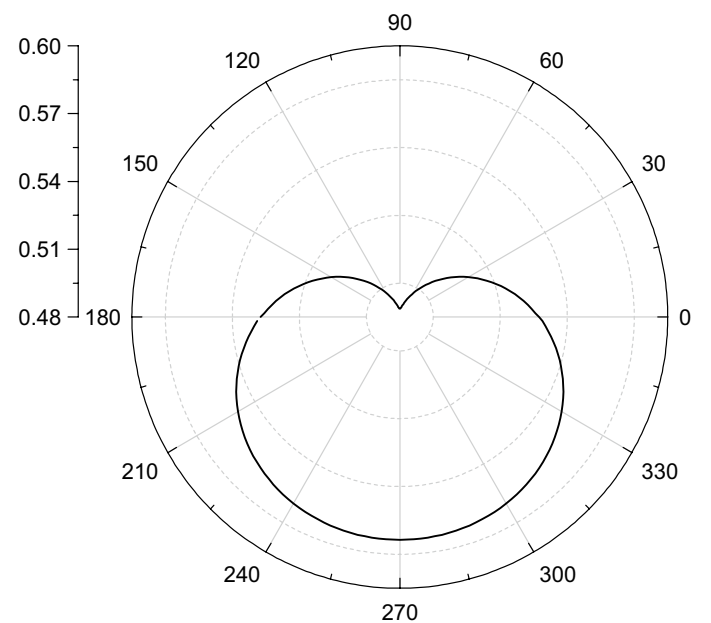

$\mathbf{a}$

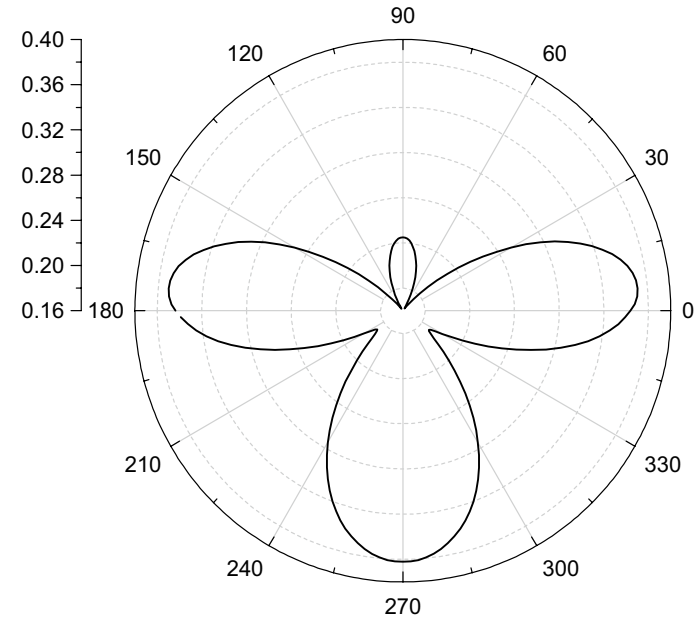

c

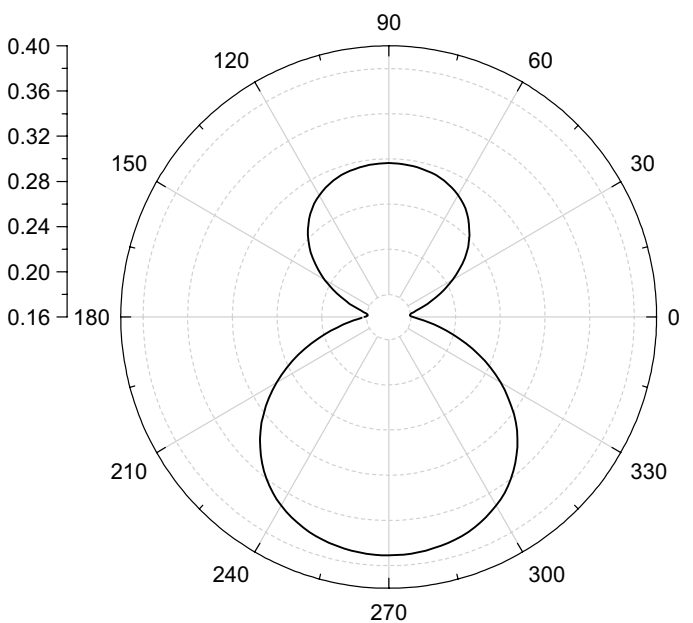

b

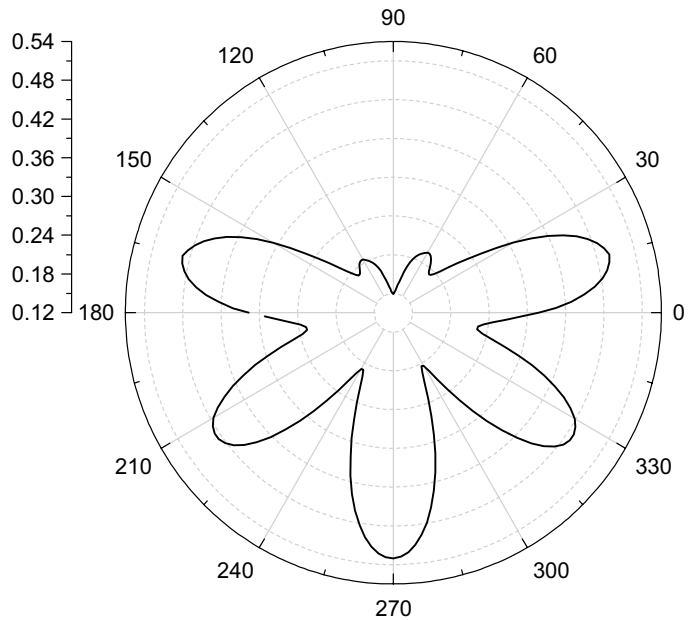

d

Figure 3 Angular distribution of scattered pressure of array plane wave versus $\mathrm{ka}(f=235 \mathrm{kHz}, 850 \mathrm{kHz}, 1109 \mathrm{kHz}$, and $2348 \mathrm{kHz})$ incident pressure amplitude is one, array number is 16 , array element width is $2.8 \mathrm{~mm}$, and array element interspace is $0.7 \mathrm{~mm}): \mathbf{a} k a=0.5, \mathbf{b} k a=1.8, \mathbf{c} k a=3.0$, and d $k a=5.0$

still in good agreement with these solutions, a result that supports the validity of this research. A noteworthy phenomenon is the greater disparity at higher frequencies, especially at sidelobe peaks.

\subsection{Simulation of Phased Arrays}

Ultrasonic phased arrays are playing an increasingly important role in acoustic detection. Detection accuracy and image visibility are sought after by researchers. Considering the complexity of the scattered field when the acoustic wave encounters obstacles in solids, it is necessary to apply the phased array principle to observe the sound fields of cylindrical scatterers in solids. Formulating an array plane wave as an incident wave is the first step in the simulations described below. Then, a Gaussian signal takes the place of the plane wave to simulate the phased array function and enable observation of the sound field. Each array element here is set linearly and equidistantly in the $2 \mathrm{D}$ model. In practical applications, the launch of a phased array system normally uses a pulse signal, and therefore a Gaussian signal is chosen because of the convenience of reducing interference and post-processing the imaging. A Gaussian wave is actually a Gaussian pulse signal. In this paper, a Gaussian wave 
consists of a sine signal and a pulse, which are subject to Gaussian distributions. Using the Gaussian pulse signal instead of a plane wave avoids confusion between scattered signals and input signals.

Figure 3 describes the angular scattered pressure distribution of the incident array plane wave with the same frequency as in Figure 2. The array plane wave is used as the incident wave, mainly to simulate the phased array, but there is no time delay among the transmitted array waves. The results shown in Figure 2 are verified in Figure 3 because the two shapes are very similar. Remarkably, the two sets of scattered pressure values are very close, except that they obviously differ at $k a=0.1$. A reasonable explanation for this phenomenon is that when the frequency is fairly low, the backscattering wave takes up a very small proportion of the space, and most of the waves propagate through the cylinder and are superimposed, leading to a greater scattered pressure amplitude in Figure 3a.

The transmitting array in a phased array has deflection and focusing functions, which ensure correct object imaging and positioning. Deflection can be achieved by changing the incident array wave. The matrix medium is excited on the top at 16 array locations using the same plane wave as the inclined pressure input, but with no time delay due to limited simulation conditions. The angular scattered pressure distributions of array plane waves with different deflection angles are shown in Figure $4 a-d$ at different frequencies during numerical wave propagation in the proposed 2D model. When the frequency is fairly low, the scattered field appears the same as before, and the influence of the incident wave angle is almost negligible (as shown in Figure 4a). The behavior of the scattered fields becomes distinctly asymmetric on both sides of the incident angle as the frequency rises. The scattered pressure amplitudes and the number of sidelobes in Figure 4d show no clear relationship, and this situation becomes worse as the frequency or incident angle increases. In fact, this phenomenon may be explained by the fact that acoustic propagation attenuation in media for different incident angles is ignored. For similar reasons, the phased array detection results are more reliable when the sample deviation is not far from the detection center.

To perform deflection and focusing simultaneously for a phased array, a Gaussian wave can be used as the transmitted signal instead of a plane wave. Previous studies indicate that when the incident wave frequency is 850 $\mathrm{kHz}$, the scattered pressure amplitude from the forward and backward directions is exactly the same (Figure $2 \mathrm{~b}$ ). The frequency of the Gaussian signal is $850 \mathrm{kHz}$, and the number of arrays is 16 . The focal point of the array beam is located at the center of the cylinder. The delay time of each transmitted signal can be calculated by the positions of the transmitted signals and the cylindrical scatterer and the signal velocity in the matrix material. Figure 5 shows the angular scattered pressure distribution of a Gaussian wave array at various instants when the Gaussian waves are deflected and focused on the surface of the cylinder.

Figure 5 shows the behavior of sound pressure amplitude when blocked by the medium. This figure provides a clearer description of the scattered field distribution on the cylinder surface from both forward and backward directions due to the time delay for Gaussian waves to propagate in the medium. The pressure distributions are clearly biased at different moments, so the directivity is therefore used to verify the correctness of using the Gaussian wave as a phased array signal. Figure 6 shows a series of collection points that are set up around the cylinder to measure sound wave directivity for a single cylinder under phased array launching. Compared with plane wave incidence, the phased array technique makes the directivity smoother, which may result from beam focusing and may be useful for contour recognition of defects.

Another noteworthy phenomenon is the mode conversion of incident longitudinal waves in solids. Figure 7 illustrates a group of numerical simulations that describe acoustic wave scattering at different moments as the incident longitudinal wave propagates into the medium with structural steel as a matrix material and aluminum as a scatterer material. The longitudinal wave field arrives at the damaged top with no interaction occurring between the two solids (as shown in Figure 7a). When the sound wave continues to travel forward and is blocked by the cylinder, interaction between the two solids occurs (as shown in Figure $7 \mathrm{~b}$ ) on the interface, resulting in complex changes in the surrounding sound field. Because of the cylinder, the incident longitudinal wave is partially scattered in the form of longitudinal waves (as shown in Figure 7c). Waveform conversion appears weak at this moment, and the wave field therefore carries the highest energy, as can be seen from the color scales representing pressure amplitude. In Figure 2d, there are obviously two kinds of waves, with the one further from the cylinder being a scattered longitudinal wave and the closer one being a scattered transverse wave that is converted by the longitudinal wave. This can be calculated and verified based on the speeds of the two kinds of waves and the time delay.

The scattering theory of a double cylinder is much more complex than that of a single cylinder. The consistency between the theoretical solution and the simulated scattered field for a single cylinder, as shown above, provides a feasible way to simulate the scattered field of 


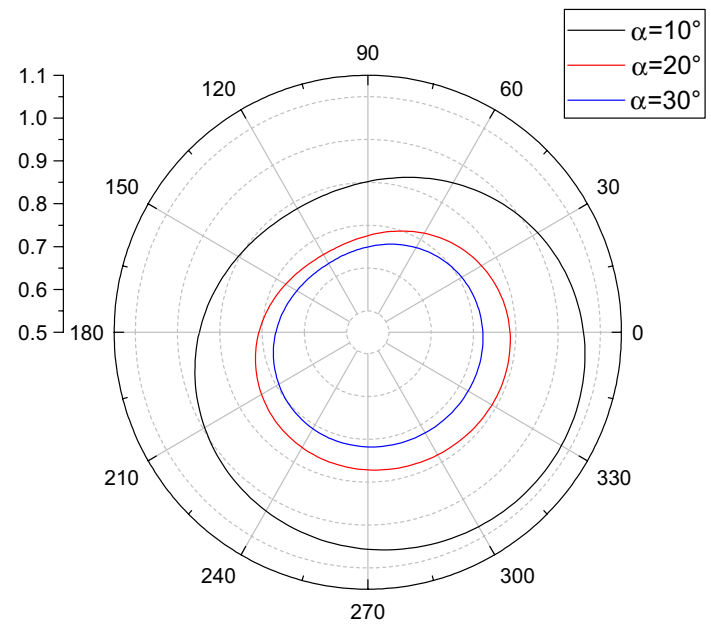

$\mathbf{a}$

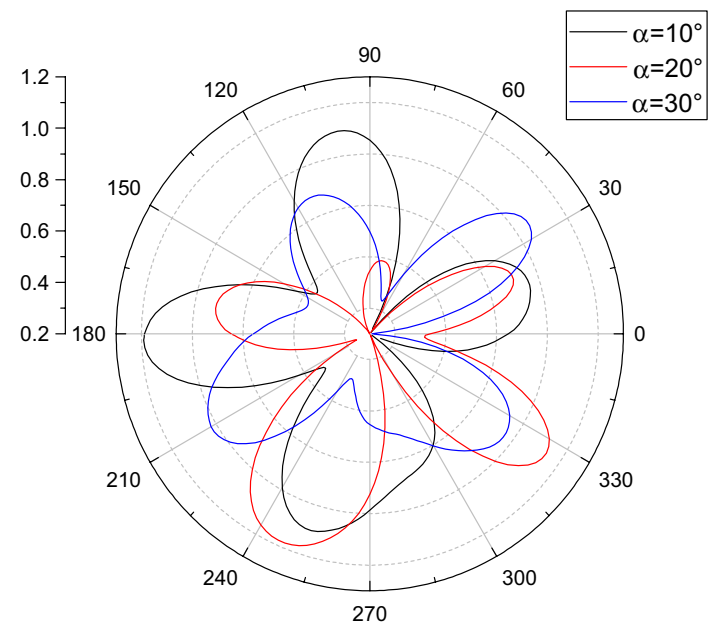

c

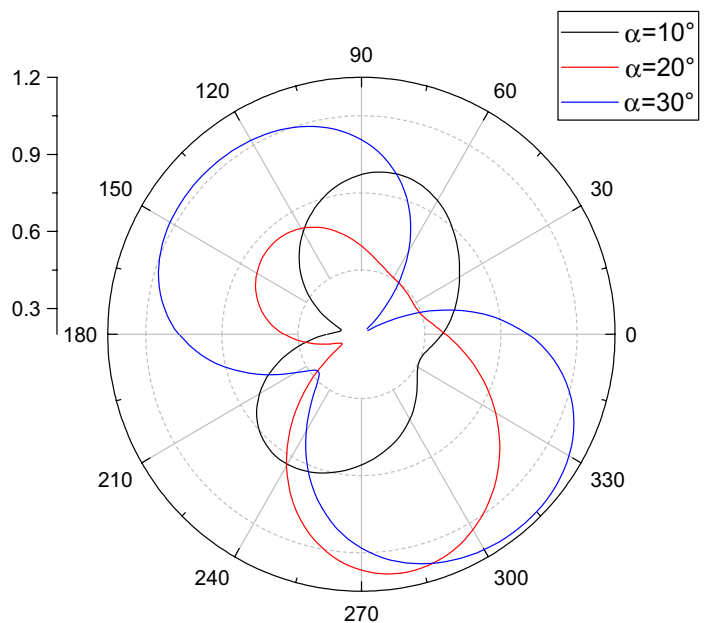

b

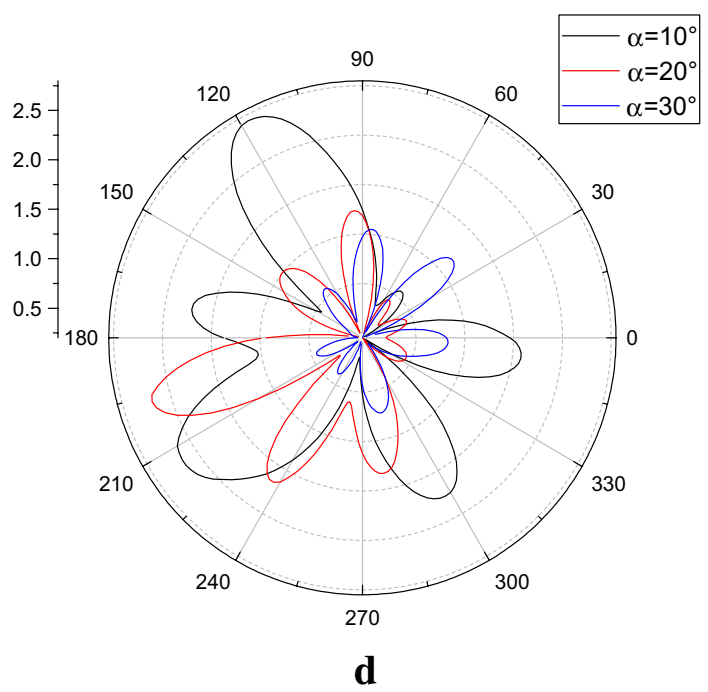

Figure 4 Angular distribution of scattered pressure of array plane wave versus $k a$ (incident angles a are $10^{\circ}, 20^{\circ}$, and $30^{\circ}$, and array number is 16 ): a $k a=0.5, \mathbf{b} k a=1.8, \mathbf{c} k a=3.0$, and $\mathbf{d} k a=5.0$

a double cylinder. The frequency of the Gaussian signal is $850 \mathrm{kHz}$, but the focal point of the array beam is not located at the center of the cylinder, but rather at the geometric center of the two cylinders. Figure 8 shows the results for the angular scattered pressure distribution of a Gaussian wave array for a double cylinder according to the distance between the two cylinders for four instants: $4.8 \mu \mathrm{s}, 5.9 \mu \mathrm{s}, 6.4 \mu \mathrm{s}$, and $7.0 \mu \mathrm{s}$.

Four sets of simulations were carried out in this research, with the distances between the centers of the two cylinders being $2,3,4$, and $6 \mathrm{~mm}$, corresponding to one to three times the radius of each cylinder. A distance of $2 \mathrm{~mm}$ means that the two cylinders are tangent and that the point of tangency is the focal point of the sound waves. When the incident waves propagate through the focal point, a portion of the scattered waves is generated near the upper half-surface of the cylinders. As the distance between the cylinders increases, backscattering becomes weaker, and the sidelobe amplitude tends to decrease (Figure 8a). As the backscattering of the scattered waves becomes weaker and the sidelobes fade, forward scattering becomes stronger (Figure $8 b$ and $c$ ). Scattered waves with low amplitudes then form around the cylinders (Figure 8d). Note that the angular scattered pressure distributions become increasingly complex with increasing distance and that this interaction can be 
Liq et al. Chin. J. Mech. Eng. $\quad$ (2019) 32:96

Page 8 of 12

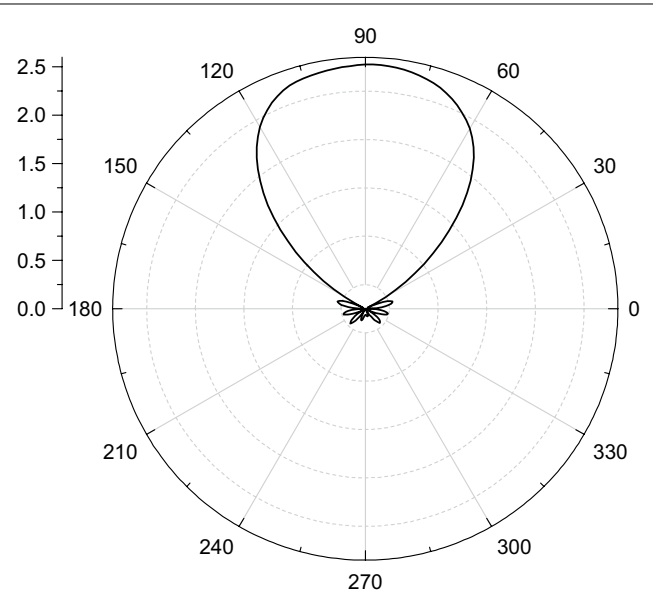

a

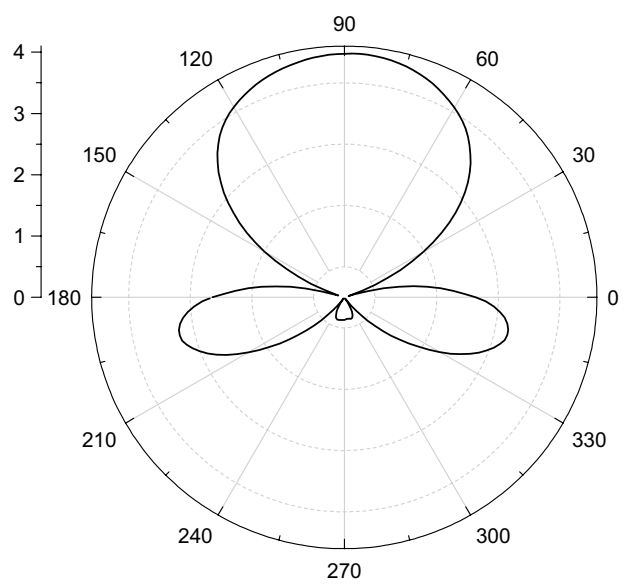

c

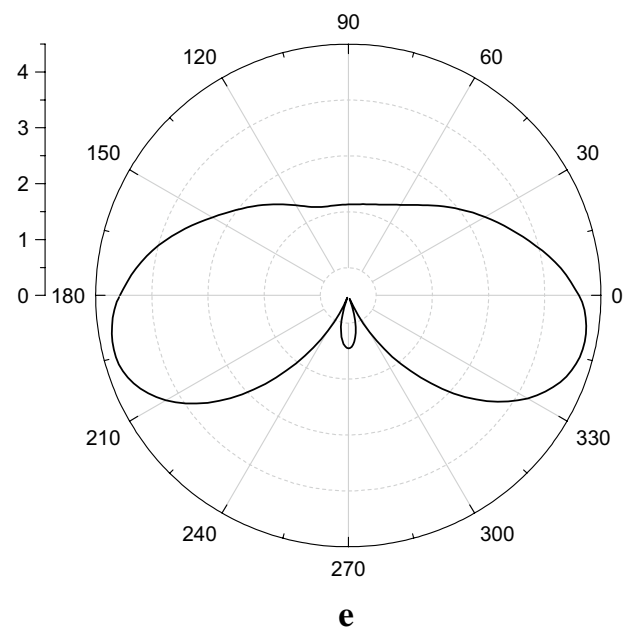

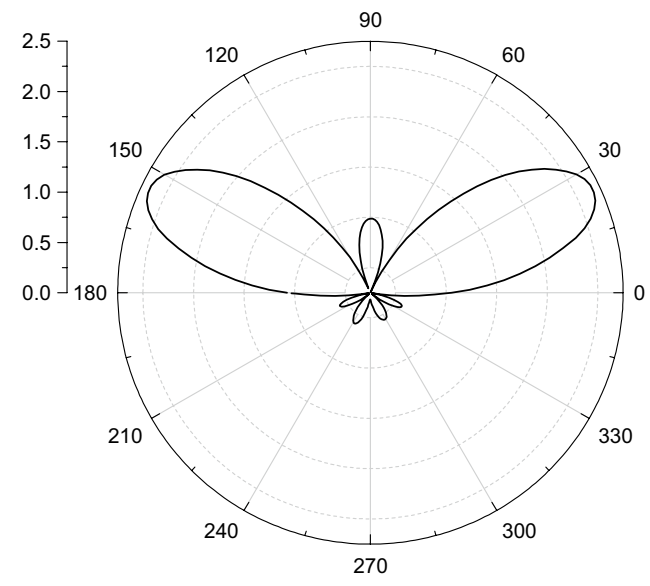

b

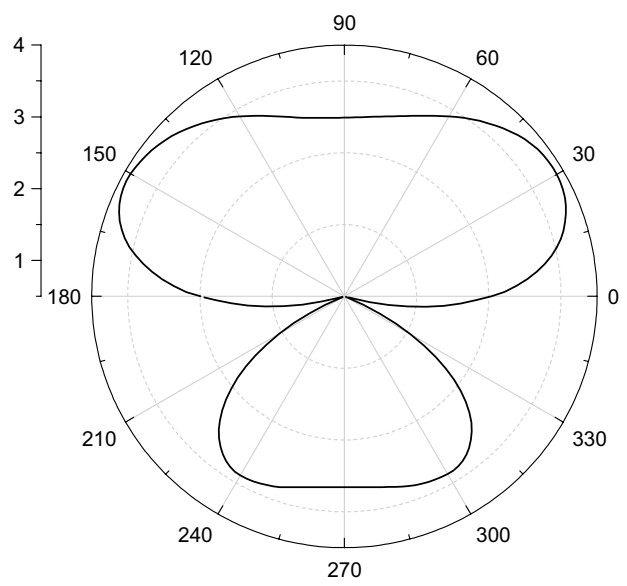

d

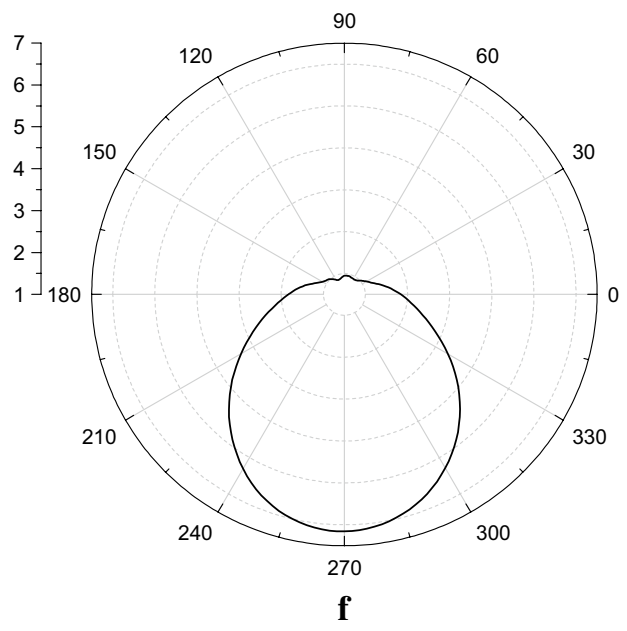

Figure 5 Pressure distribution of the ultrasonic field for a single cylinder at various instants (impulse signal amplitude is $20, f=850 \mathrm{kHz}$ ): a $t=4.2 \mu \mathrm{s}, \mathbf{b} t=4.2 \mu \mathrm{s}, \mathbf{c} t=4.6 \mu \mathrm{s}, \mathbf{d} t=4.8 \mu \mathrm{s}, \mathbf{e} t=5.0 \mu \mathrm{s}$, and $\mathbf{f} t=5.2 \mu \mathrm{s}$ 


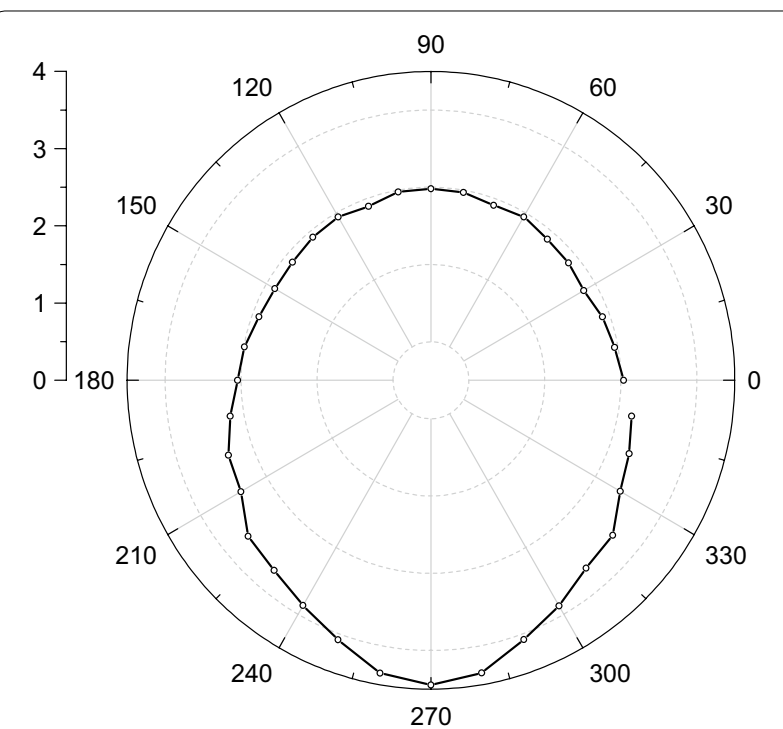

Figure 6 Directivity of sound wave for a single cylinder with phased array technique $(f=850 \mathrm{kHz}$, and $\mathrm{ka}=1.8)$

neglected when the distance is over three times the cylinder radius.

Figure 9a shows the relative distances between two cylinders and the phase array, and a conclusion can be drawn from Figure $9 \mathrm{~b}$ that the directivity becomes more concentrated, and the intensity of backscattering is stronger than that of forward scattering. Unlike the situation shown in Figure 7 and Figure 2b, directivity becomes sharper, and backscattering becomes stronger than forward scattering in the case of a double cylinder. As the distance between cylinders increases, scattering becomes stronger, but the magnitude of the increase is finite, especially for backscattering.

\section{Conclusions}

The investigations reported in this paper led to the following conclusions:

The theoretical solutions based on scattering theory by a plane wave were elucidated, especially in Figure 2, and they are in good agreement with the simulation results obtained here. Similar results were also obtained using Gaussian wave incidence. All these observations illustrate that the proposed mechanism is correct and meaningful.

The angular scattered pressure distributions for single and double cylinders were studied using the phased array method. For a single cylinder, the scattered field directivity is relatively uniform in the circumferential direction. For a double cylinder, the scattered field distribution has particular shapes at different moments or distances, and the directivity becomes intense along the incident direction. Outside a certain distance, the waveform is very complex, and the interaction between cylinders becomes weaker.

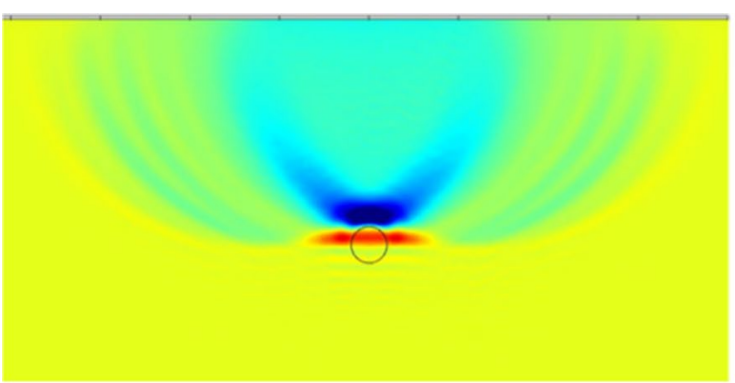

a

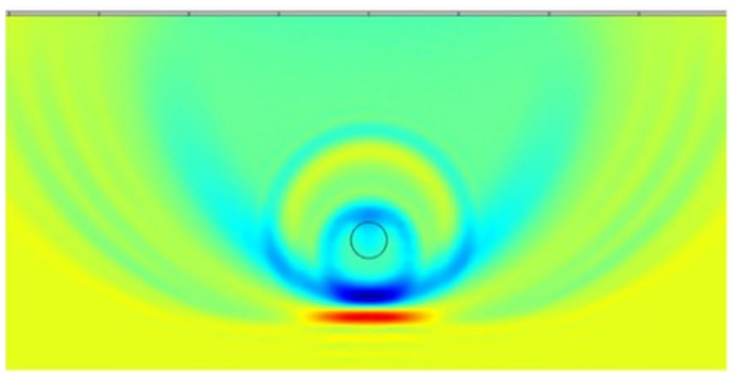

c

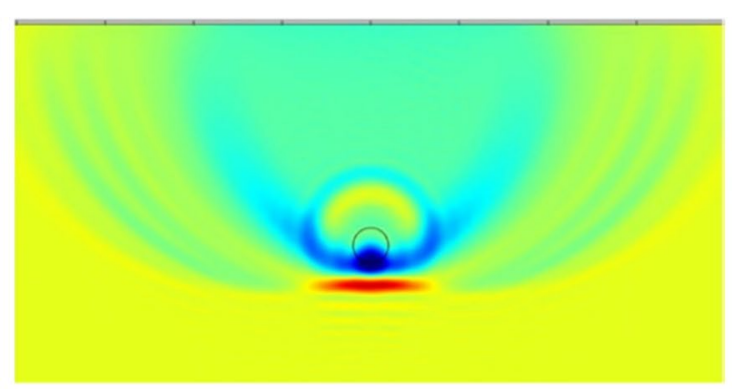

b

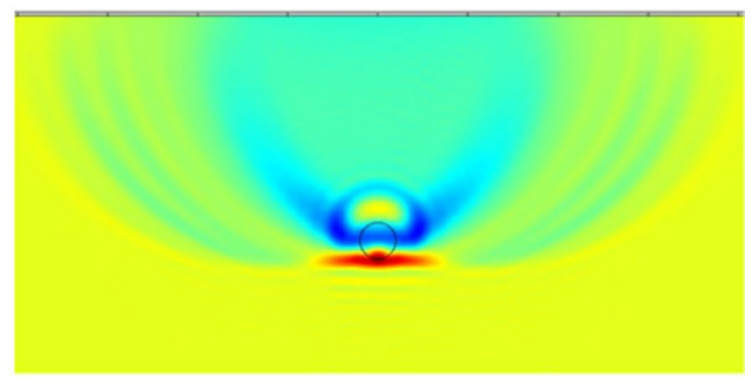

d

Figure 7 Snapshots of the scattered wave filed at different moments using Gaussian waves, and mode conversion occurs when acoustic waves are scattered by a cylinder: $\mathbf{a} t=4.4 \mu \mathrm{s}, \mathbf{b} t=4.9 \mu \mathrm{s}, \mathbf{c} t=5.3 \mu \mathrm{s}$, and $\mathbf{d} t=6.0 \mu \mathrm{s}$ 


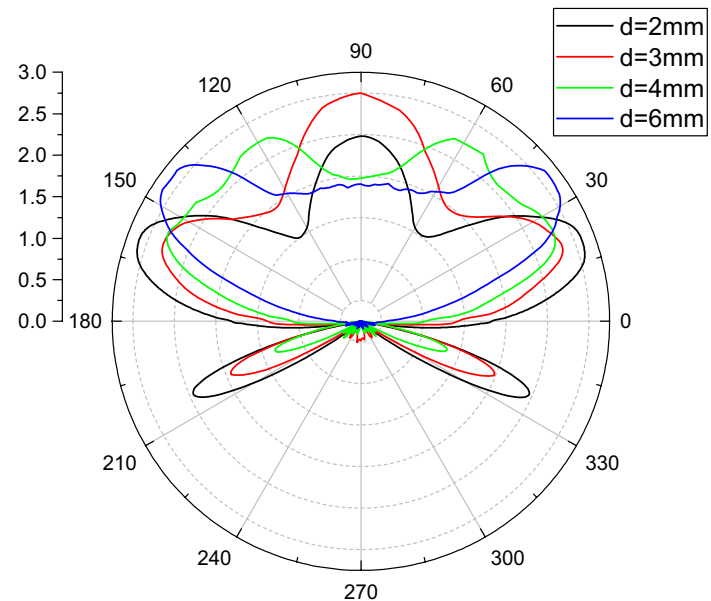

a

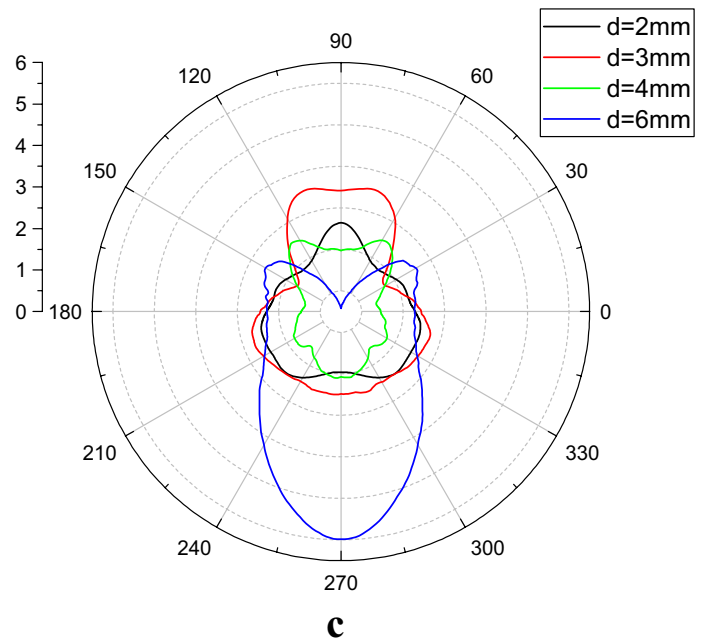

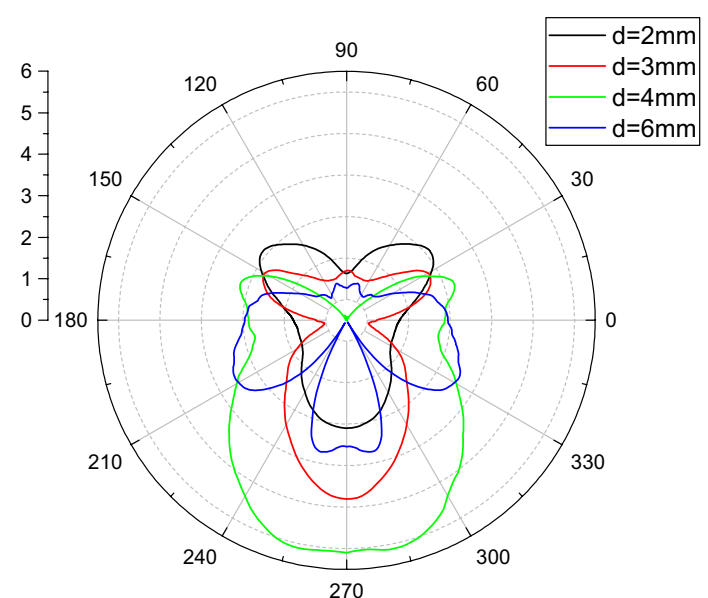

b

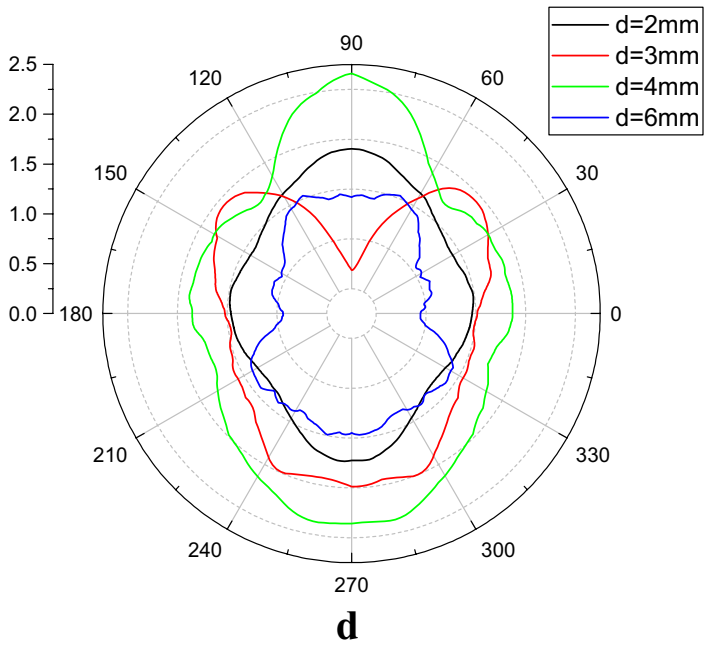

Figure 8 Pressure distributions of the ultrasonic wave propagating at various instants, which show the scattered filed amplitude behavior when the distances between two cylinders are $2,3,4$, and $6 \mathrm{~mm}: \mathbf{a} t=4.8 \mu \mathrm{s}, \mathbf{b} t=5.9 \mu \mathrm{s}, \mathbf{c} t=6.4 \mu \mathrm{s}$, and $\mathbf{d} t=7.0 \mu \mathrm{s}$, respectively

Phased array technique
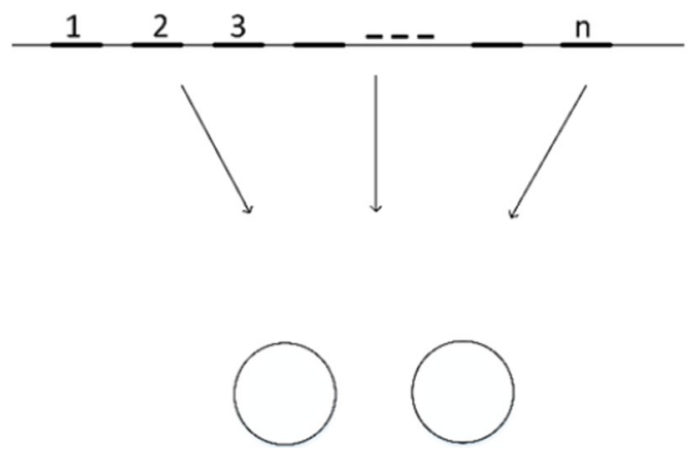

a

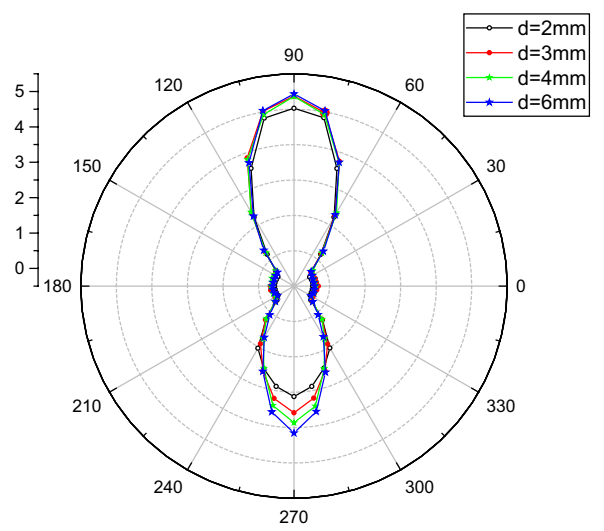

b

Figure 9 Location and directivity of sound wave with phased array technique versus different distances for cylinders $(f=850 \mathrm{kHz}$, and ka=1.8) 
Future work will be oriented towards investigating the scattered field of double cylinders under various conditions, continuing the work reported in this paper. In fact, many factors affect the accuracy and imaging quality of phased array synthetic acoustic field experiments, such as inhomogeneous sample material or complex defect shape. These factors will bring more challenges to the research. Experimentally combining a phased array imaging system with data processing of the scattered field distribution will promote quantitative analysis of defects and uniform imaging quality.

\section{Authors' Contributions}

$\mathrm{XL}$ was in charge of the whole trial; JM and XL wrote the manuscript; HW, SG, $J L$ and $Y L$ assisted with numerical analyses. All authors read and approved the final manuscript.

\section{Authors' Information}

Xiaozhou Liu, born in 1966, is currently a professor at Nanjing University, China. He received his PhD degree from Nanjing University, China in 1999. His research interests include nonlinear acoustics, ultrasonic non-destructive testing and medical ultrasound.

Jian Ma, born in 1992, is currently an engineer at AAC Technologies, China. He received his master degree on Acoustics in Nanjing University, China, in 2018.

Haibing Wang, born in 1978, is currently a PhD candidate at Nanjing University, China.

Sha Gao, born in 1992, is currently an engineer at AAC Technologies, China. He received his master degree on Acoustics in Nanjing University, China, in 2018.

Yifeng Li, born in 1976, is currently a professor at Nanjing University of technology, China. His main research interests include nonlinear acoustics.

Jiehui Liu, born in 1973, is currently an associated professor at Nanjing University, China. He received his PhD degree from Nanjing University, China, in 2000. His research interests include nonlinear acoustics and medical ultrasound.

\section{Competing Interests}

The authors declare that they have no competing interests.

\section{Funding}

Supported by National Key R\&D Program of China (Grant No

2016YFF0203000), State Key Program of National Natural Science Foundation of China (Grant No. 11834008), National Natural Science Foundation of China (Grant Nos. 11774167, 61571222), Fundamental research funds for the Central Universities (Grant No. 020414380001), State Key Laboratory of Acoustics, Chinese Academy of Science (Grant No. SKLA201809), Key Laboratory of Underwater Acoustic Environment, Chinese Academy of Sciences (Grant No. SSHJ-KFKT-1701), AQSIQ technology R\&D program (Grant No. 2017QK125), and Innovative Talents Program of Far East NDT New Technology \& Application Forum.

\section{Author Details \\ ${ }^{1}$ Key Laboratory of Modern Acoustics, Institute of Acoustics and School of Physics, Nanjing University, Nanjing 210093, China. ${ }^{2}$ College of Computer Science and Technology, Nanjing University of Technology, Nanjing 211800,} China.

Received: 19 April 2019 Revised: 10 October 2019 Accepted: 19 November 2019

Published online: 29 November 2019

\section{References}

[1] J J Faran. Sound scattering by solid cylinders and spheres. The Journal of the Acoustical Society of America, 1951, 23(4): 405-418.

[2] N D Veksler. The analysis of peripheral waves in the problem of plane acoustic pressure wave scattering by a circular cylindrical shell. Acta Acustica United with Acustica, 1989, 69(2): 63-72.

[3] A Boström, T Kundu. Axisymmetric scattering of a plane longitudinal wave by a circular crack in a transversely isotropic solid. Journal of Applied Mechanics, 1991, 58(3): 695-702.

[4] VW Lee. A note on the scattering of elastic plane waves by a hemispherical canyon. International Journal of Soil Dynamics and Earthquake Engineering, 1982, 1(3): 122-129.

[5] N G Einspruch, R Truell. Scattering of a plane longitudinal wave by a spherical fluid obstacle in an elastic medium. Journal of the Acoustical Society of America, 1960, 32(2): 214-220.

[6] A Taflove, M E Brodwin. Numerical solution of steady-state electromagnetic scattering problems using the time-dependent Maxwell's equations. IEEE Transactions on Microwave Theory and Techniques, 1975, 23: 623-630.

[7] W G Neubauer, R H Vogt, L R Dragonette. Acoustic reflection from elastic spheres, I. Steady-state signals. Journal of the Acoustical Society of America, 1974, 55(6): 1123-1129.

[8] A Capelli, J C Kapil, I Reiweger, et al. Speed and attenuation of acoustic waves in snow: Laboratory experiments and modeling with Biot's theory. Cold Regions Science and Technology, 2016, 125: 1-11.

[9] R C Addison, A N Sinclair. Calculated and measured ultrasonic response of an elastic cylinder embedded in an elastic medium. Review of Progress in Quantitative Nondestructive Evaluation, 1992, 11a: 105-111.

[10] R M White. Elastic wave scattering at a cylindrical discontinuity in a solid. Journal of the Acoustical Society of America, 1958, 30(8): 771-785.

[11] M A Biot. Theory of propagation of elastic waves in a fluid-saturated porous solid I Low-frequency range. Journal of the Acoustical Society of America, 1956, 28(2): 168-178.

[12] M A Biot. Theory of propagation of elastic waves in a fluid-saturated porous solid. II. Higher frequency range. Journal of the Acoustical Society of America, 1956, 28(2): 179-191.

[13] N P Chotiros. Biot model of sound propagation in water-saturated sand. Journal of the Acoustical Society of America, 1995, 97(1): 199-214.

[14] M A Biot. Mechanics of deformation and acoustic propagation in porous media. Journal of Applied Physics, 1962, 33(4): 1482-1498.

[15] Y H Pao, C C Mow, J D Achenbach. Diffraction of elastic waves and dynamic stress concentrations. Journal of Applied Mechanics, 1973, 40(4): 872.

[16] M Sato, N A Hall, Y Takahata, et al. Expression of Acoustic Fields in Solids by Scalar and Vector Velocity Potentials, Atlanta, USA: Ultrasonics Symposium. IEEE, 2001 [2001-10-7]. https://ieeexplore.ieee.org/document/99185 3.

[17] Y M Kim, B H Lee, J G Ih. Scattering of longitudinal waves by a cylindrical cavity in an attenuating solid. Journal of the Acoustical Society of America, 1993, 93(1): 93-101.

[18] P Beattie, R C Chivers, LW Anson. Ultrasonic backscattering from solid cylindrical inclusions in solid elastic matrices: A comparison of theory and experiment. Journal of the Acoustical Society of America, 1993, 94(6): $3421-3427$

[19] S I Rokhlin, W Huang, Y C Chu. Ultrasonic scattering and velocity methods for characterization of fibre-matrix interphases. Ultrasonics, 1995, 33(5): 351-364.

[20] W Huang, S I Rokhlin, Y J Wang. Effect of fibre-matrix interphase on wave propagation along, and scattering from, multilayered fibres in composites. Transfer matrix approach. Ultrasonics, 1995, 33(5): 365-375.

[21] C F Ying, RTruell. Scattering of a plane longitudinal wave by a spherical obstacle in an isotropically elastic solid. Journal of Applied Physics, 1956, 27(9): 1086-1097.

[22] L Flax, G C Gaunaurd, H Uberall. Theory of resonance scattering. Physical Acoustics, 1981, 15: 191-294.

[23] J P Sessarego, J Sageloli, R Guillermin, et al. Scattering by an elastic sphere embedded in an elastic isotropic medium. Journal of the Acoustical Society of America, 1998, 104(5): 2836-2844.

[24] P Olsson, S K Datta, A Boström. Elastodynamic scattering from inclusions surrounded by thin interface layers. Journal of Applied Mechanics, 1990, 57: 672-676. 
[25] G Johnson, R Truell. Numerical computations of elastic scattering cross sections. Journal of Applied Physics, 1965, 36(11): 3466-3475.

[26] Y Iwashimizu. Scattering of elastic waves by a movable rigid sphere embedded in an infinite elastic solid. Journal of Sound and Vibration, 1972, 21(4): 463-469.

[27] D L Jain, R P Kanwal. Scattering of P and S waves by spherical inclusions and cavities. Journal of Sound and Vibration, 1978, 57(2): 171-202.

[28] R J McBride, D W Kraft. Scattering of a transverse elastic wave by an elastic sphere in a solid medium. Journal of Applied Physics, 1972, 43(12): 4853-4861.

[29] R Hiptmair, L Scarabosio, C Schillings. Large deformation shape uncertainty quantification in acoustic scattering. Advances in Computational Mathematics, 2018, 44(4): 1-44.

[30] E Johannes, G H Hu. Acoustic scattering from corners, edges and circular cones. Archive for Rational Mechanics and Analysis, 2018, 228(2): 653-690.

[31] G Bao, G H Hu, TYin. Time-harmonic acoustic scattering from locally perturbed half-planes. SIAM Journal on Applied Mathematics, 2018, 78(5): 2672-2691.

[32] KTahsin, X Antoine, S P A Bordas. B-spline FEM for time-harmonic acoustic scattering and propagation. Journal of Theoretical and Computational Acoustics, 2018, 26(4): 1850059.

[33] N Kaina, F Lemoult, M Fink, et al. Negative refractive index and acoustic superlens from multiple scattering in single negative metamaterials. Nature, 2015, 525(7567): 77-81.
[34] L Ayton. Acoustic scattering by a finite rigid plate with a poroelastic extension. Journal of Fluid Mechanics, 2016, 791: 414-438.

[35] S J Jin, X L Yang, S L Chen, et al. Development and application of ultrasonic phased array inspection technology. Journal of Electronic Measurement and Instrumentation, 2014, 9: 925-934.

[36] J Zhang, B W Drinkwater, P D Wilcox. Effects of array transducer inconsistencies on total focusing method imaging performance. NDT and $E$ International, 2011, 44(4): 361-368.

[37] J P Jiao, Y Chang, X R Sun, et al. Study on the identification of the direction of the crack with vector total focusing method in ultrasonic phased arrays and the influence parameters. Journal of Nanjing University (Natural Sciences), 2015, 51(11) Supplement: 72-77.

[38] Y F Zhou, L Ma. Sound scattering by fluid-saturated porous cylinders. Acta Physica Sinica, 2000, 49(3): 480-486.

[39] J Chu, Q B Han. Acoustic scattering by cylinders immersed in a fluid medium: Modeling, solving and verifying. Modern Physics Letters B, 2018, 32(34n36): 1840101.

[40] X Li, J Bao, Y Huang, et al. Traveling wave excitation sources for FEM analysis of scattering in acoustic waveguide. Microsystem Technologies, 2019, 25(7): 2783-2792.

\section{Submit your manuscript to a SpringerOpen ${ }^{\circ}$ journal and benefit from:}

- Convenient online submission

$\checkmark$ Rigorous peer review

- Open access: articles freely available online

- High visibility within the field

- Retaining the copyright to your article

Submit your next manuscript at $\boldsymbol{\nabla}$ springeropen.com 